\title{
Alter
}

Revue de phénoménologie

$27 \mid 2019$

Patočka

\section{Précarité ontologique et consistance des êtres}

\section{Pierre Montebello}

\section{OpenEdition}

Journals

Édition électronique

URL : https://journals.openedition.org/alter/1918

DOI : $10.4000 /$ alter.1918

ISSN : 2558-7927

\section{Éditeur :}

Association ALTER, Archives Husserl (CNRS-UMR 8547)

\section{Édition imprimée}

Date de publication : 1 novembre 2019

Pagination : 189-209

ISBN : 978-2-9550449-5-7

ISSN : 1249-8947

\section{Référence électronique}

Pierre Montebello, «Précarité ontologique et consistance des êtres », Alter [En ligne], 27 | 2019, mis en ligne le 22 décembre 2020, consulté le 13 juin 2021. URL : http://journals.openedition.org/alter/1918 : DOI : https://doi.org/10.4000/alter.1918

Ce document a été généré automatiquement le 13 juin 2021

Revue Alter 


\title{
Précarité ontologique et consistance des êtres
}

\author{
Pierre Montebello
}

\section{De la précarité ontologique}

1 C'est un fait nouveau, la précarité hante nos discours. Il est non seulement manifeste que le mot précaire est très répandu aujourd'hui, que son usage s'est multiplié dans les champs éthiques, économiques, sociaux, écologiques, mais il est plus notable encore que notre perception des choses a opéré, en arrière-fond, une mutation profonde. Avant que ce mot ne s'impose, l'Antiquité parlait de la faiblesse humaine comme signe de son manque de constance ou de force face au destin, le christianisme faisait fond sur la fragilité humaine (la fragilitas de saint Augustin, structure plus positive où se dévoile que notre seul salut est d'accepter notre fragilité devant Dieu $^{1}$.) On parle aujourd'hui de précarité pour désigner quasiment tous les champs de la réalité.

2 Nous voudrions montrer que cette mutation porte d'abord sur ce que être veut dire. C'est un autre sentiment qui apparaît, le sentiment que la précarité est consubstantielle, si l'on peut dire, à l'être, la conviction que c'est l'être lui-même qui est précaire. L'être est précaire parce qu'il est être, pour aucune autre raison destinale ou divine. Au-delà de la faiblesse anthropologique et théologique qui fut l'horizon commun de la pensée occidentale, la précarité installe son ordre et son désordre au niveau le plus large, celui de l'être. C'est de ce rapport à l'universalité des choses dont il va être question. De la même manière que Foucault parlait de ruptures de perception dans le temps historique, nous allons essayer de saisir le moment où la perception des êtres change, de circonscrire les raisons de la mutation du regard qui fera que les êtres vont globalement nous apparaître comme étant insuffisants, précaires. Nous associons à la vulnérabilité le fait de pouvoir être blessé, diminué dans la puissance de vie, dans la capacité vitale. L'usage étendu, social et politique, de la caractérisation «vulnérable » dans la théorie du care de Joan Tronto ne modifie pas ce sens premier: à la vulnérabilité physique s'adjoint une vulnérabilité sociale et relationnelle qui réclame 
soins et attention en dépassant la répartition des genre ${ }^{2}$. Marie Garrau, dans Politiques de la vulnérabilité, peut ainsi décrire la vulnérabilité comme " une structure commune d'existence » où la dimension sociale relationnelle est dominante ${ }^{3}$. Nous nommons plus généralement "précarité » non pas une structure d'existence sociale et politique mais une structure d'être telle que l'être ne consiste qu'en ces relations, sans posséder aucune autosuffisance. Cette ontologie relationnelle a été l'apport majeur de la philosophie post-kantienne, de Nietzsche à Bergson, de William James à Whitehead ${ }^{4}$. Une partie de la modernité se tient là, dans un rapport inédit entre être et précarité : l'être est précaire, et l'être est précaire parce qu'il est, sans aucune autre raison, parce qu'il ne consiste que dans des relations qui peuvent à tout moment se défaire. De cette précarité de l'être, il a peu été dit. Or, c'est bien l'élargissement de la précarité vers l'ensemble des êtres qui s'est imposé comme fait majeur.

Elle a eu pour première condition une modification radicale de notre perception de la nature. Peut-être même est-ce le point le plus décisif. Les modernes ne sont pas seulement ceux qui ont réduit la nature à l'indifférence physicaliste, ils sont ceux qui ont aussi acquis progressivement la certitude que la nature est elle aussi vulnérable. Ce sentiment prendra du temps à s'installer car toute la pensée antique et médiévale, renaissante et moderne de la nature lui est opposée. C'est seulement au $\mathrm{XX}^{\mathrm{e}}$ siècle qu'il devient clair que la nature n'a pas les propriétés qu'on lui attribuait en général. Ce sera le premier pas vers une pensée générale de la précarité ontologique.

Nous n'allons pas parcourir toute les époques de la pensée de la nature mais nous situer à l'orée de l'anthropocène, à la fin du XVIII siècle. À ce moment où s'invente un genre nouveau, la littérature catastrophiste, promise à un bel avenir. Un nouveau type de récit se met à englober homme et nature, à associer l'histoire de la nature et l'histoire du genre humain. Sur quoi s'appuie-t-il ? Sur la catastrophe. La catastrophe fait son apparition comme concept central pour articuler l'histoire de la nature et l'histoire du genre humain. La nature n'est plus seulement perçue comme indifférente à cause de ses lois impassibles, elle est vue comme violente, source de l'impossibilité de faire monde, désastre des mondes humains, vulnérabilité des mondes. On se met à penser que la catastrophe n'est pas un événement remarquable, une rupture dans le cours paisible des choses, mais qu'elle a été l'état naturel de la nature aux premiers temps, l'événement majeur de l'histoire naturelle à ses origines. La nature va alors désigner l'anti-monde, l'anti-humain. Impossible de faire monde pour les humains en ces temps originaires désolés, assez proches de nous tout de même (l'origine de la Terre pour le $\mathrm{XVIII}^{\mathrm{e}}$ siècle n'est pas si lointaine). Ce que la nature originaire porte en elle sans répit, ce sont les « ruines du monde».

5 Toute catastrophe présente nous rappelle les temps originaires de la nature, réveille en nous le sentiment d'un désordre radical, d'anomalies, de convulsions. Buffon le dit, dans Les époques de la nature: "Les premiers hommes témoins des mouvements convulsifs de la nature de la terre, encore récents et très fréquents ", animés par une "terreur funeste ", vont chercher à s'unir pour trouver moyen d'habiter la Terre. Il évoque ce "moment » où « la Terre est devenue le domaine de l'homme», car la Terre domestiquée ne fut pas toujours. Nous conservons le souvenir de ces «malheurs du monde", ravages, volcans, inondations, gouffres et secousses, nous gardons en mémoire ces catastrophes originaire ${ }^{5}$. Les mythologies, les traditions sont des souvenirs du déluge et des convulsions de la terre. 
6 Nous comprenons en somme que la terre humanisée n'a pas toujours été, bien au contraire, la nature représente la terre déshumanisée, sans mondes. Dans ses Recherches sur l'origine du despotisme oriental, Nicolas-Antoine Boulanger raconte lui aussi l'histoire "de l'homme échappé de la ruine du monde ${ }^{6}$. Il brosse le même tableau de ce passé violent de la nature. Se souvenir du passé de la nature, c'est prendre conscience qu'il y eût des « temps déplorables » scandés par les révolutions naturelles et les catastrophes, où tout n'était que ténèbres, incendies, tremblements, inondations, où l'homme errait dans les ruines du monde, c'est se remémorer des temps où "l'homme s'est regardé comme étant l'objet de la haine et de la vengeance de toute la nature irritée ; toutes les sociétés ont été rompues ; les hommes ont été obligés d'errer à l'aventure dans les ruines du monde, au gré de tous les fléaux qui semblaient les poursuivre ${ }^{7}$. Si l'on peut dire que la nature a ses " monuments ", c'est au sens où ses anciennes catastrophes, ses désastres passés, racontent les «malheurs du monde ». Quand bien même, l'homme oppose toute sa force morale à la nature pour édifier des mondes, les monuments de la nature, à savoir ses anciennes « révolutions", lui rappellent «l'inconstance et la fragilité » des mondes humains ${ }^{8}$.

7 Cette vision d'une puissance naturelle démesurée et indomptable, voire catastrophique, subsistera comme un inconscient de la pensée et de l'imagination de la nature, elle traversera les époques. Mais dans nos schémas les plus contemporains, la nature ne peut plus relever de cette seule puissance entière. Avec l'anthropocène, s'impose l'idée précisément inverse que la ruine du monde n'est pas engendrée par la force de la nature, mais par sa faiblesse, par son incapacité à juguler la démesure humaine.

8 En lisant l'Antigone de Sophocle dans son Principe de responsabilité, Hans Jonas nous livre une réflexion dont on peut faire l'emblème d'une prise de conscience d'un statut nouveau de la nature: sa vulnérabilité. On se souvient que dans un chant fameux, Sophocle célèbre la puissance ingénieuse de l'homme, "merveille des merveilles ", mais ce même chant dit aussi que l'homme ne saurait jamais diminuer la puissance de la nature. L'homme a beau tourmenter « la déesse auguste entre toutes, la Terre, la Terre éternelle et infatigable, avec ses charrues ", qui songerait à dire, dans l'Antiquité, que l'homme épuise la Terre, qu'il entame en quoi que ce soit sa force fécondante ? Ni les labours, ni l'élevage, ni la domestication, n'affectent l'alme nature. L'agir humain laisse «inchangée la nature englobante des règnes" naturels, eau, mer, terre, vie, comme le rappelle Hans Jonas. Les violences humaines ne « diminuent pas leurs forces créatrices ${ }^{9}$.

9 Hans Jonas ne s'attarde pas ici sur un terme essentiel : la nature est infatigable, elle est inépuisable littéralement, sa force reste intacte, et "sans âge ». Cela signifie que la nature accueille la violence humaine ponctuelle et locale en préservant immuablement son ordre cosmique. Le plus important ici n'est pas de manifester l'immutabilité de la nature, de dire son fond invariant, c'est de souligner l'impossibilité de diminuer en quoi que ce soit la force entière de la nature par quelque action que ce soit. Si la nature est une puissance immuable, c'est parce que ses forces créatrices sont impérissables, à jamais inentamées. Nous voulons dire que épuisement et nature sont des termes antinomiques dans ce chant, la nature est précisément ce qui ne peut jamais être épuisé. C'est pourquoi agir et création humaine ne sont pas logés à la même enseigne. Les hommes, malgré leur ingéniosité, sont soumis au changement, ils ne sont pas immuables, ils affrontent le contingent et le destin, la résistance des choses, la corruption, la fatigue, l'épuisement. La nature inépuisable garantit même en retour la 
stabilité de la condition humaine. Quels que soient les effets de son artifice et de sa liberté, rien ne bouleverse en effet les normes de l'existence humaine, car il y a comme un lien de nature entre inconsistance du destin humain et consistance de la condition humaine :

Et, même, c'est précisément l'inconsistance du destin humain qui garantit la consistance de la condition humaine. Le hasard, la chance, la stupidité, les grands compensateurs dans les affaires humaines, agissent comme une sorte d'entropie et

finissent par faire converger tous les projets déterminés vers la norme éternelle ${ }^{10}$.

On comprend que la question éthique centrale de l'Antiquité ait été de se demander comment être constant et consistant face aux aléas de la fortune. C'est ainsi que la sphère de la responsabilité est restée bornée à une sphère humaine. Il n'y avait pas de sentiment de responsabilité envers la nature fondamentalement considérée comme un ordre inaffecté par l'agir humain. La nature était cette totalité insensible à l'action locale, cette puissance irréductible à l'agir de ses agents.

11 Cette « immutabilité essentielle de la nature en tant qu'ordre cosmique », pour parler avec Hans Jonas, s'accompagne d'un régime de perception dont il faut dire le sens. Pour l'Antiquité, la nature est au sens propre, invulnérable, elle n'est défaite par rien, sa puissance ne peut jamais être diminuée ou affectée par l'agir humain. Si la responsabilité humaine ne porte pas sur la nature, même dans le déploiement de l'action technique, c'est que cette action n'est pas capable d'entamer la nature, en quelque manière que ce soit, ni en affectant son fond, ni en diminuant ou épuisant sa puissance. Pour cette raison, "la nature ne fut pas un objet de la responsabilité humaine ", dit Hans Jonas. La responsabilité concernait seulement ce qui peut être épuisé, anéanti, rendu inconsistant, à savoir l'homme. En revanche, la nature, comme plan de consistance, retour universel et cyclique d'une activité normative et réglée, n'avait nul besoin qu'on s'occupe d'elle, qu'on la prenne en charge.

Il n'est pas injustifié de parler alors avec Hans Jonas d'une éthique "anthropocentrique ». En effet, la sphère de la responsabilité antique était extranaturelle. Ou ce qui revient au même, elle était strictement interhumaine, elle concernait cité et polis. Ainsi s'explique que la pensée de la nature ait été l'objet d'une position paradoxale dans l'Antiquité. Elle sera l'idéal de l'éthique (la nature comme mesure de l'homme), du fait même de son ordre et de sa permanence, mais elle ne sera pas l'objet de l'éthique puisqu'aucune action ne la vise en tant que telle. Nombre de traités antiques ont pour norme la nature, ils enseignent à vivre selon la nature, sans que la nature ne suscite la moindre obligation par elle-même. Pour que l'éthique porte sur la nature, comme objet d'obligation et d'impératifs, qu'elle soit cosmocentrique, il faudra que la nature change de signification, il faudra qu'elle revête un aspect tout à fait neuf et absolument opposé à sa puissance et autosuffisance antique: la vulnérabilité et non plus « l'invulnérabilité d'un ensemble dont les profondeurs ne sont pas perturbées par l'importunité humaine $\aleph^{11}$. Une bonne part de l'éthique contemporaine a pris en charge l'homo vulnerabilis, mais tout autre chose est de penser le surgissement de cette perception nouvelle, la natura vulnerabilis.

\section{La natura vulnerabilis}

13 Attardons-nous sur le sens de ce surgissement et de cette perception nouvelle. On trouve mention de la «nostra natura vulnerabilis » dans les textes latins (de Roberti 
Bellarmini par exemple), mais pas de la natura vulnerabilis en elle-même. Et pour cause. Nous l'avons dit, la vulnérabilité concerne étymologiquement le fait de pouvoir être blessé, atteint, contraint, restreint. Tandis que la fragilité nomme ce qui peut être brisé, cassé. L'anthropologie chrétienne fera fond sur la fragilité comme structure même de l'existence humaine face à un Dieu qui retient en lui toute la puissance d'être ${ }^{12}$. Tandis que le fait d'être blessé concerne plus généralement le vivant et son manque de protection face aux événements.

Dans l'Antiquité, à la différence des dieux, les êtres vivants prêtent le flanc au tranchant de qui arrive, à la blessure des événements ou du destin. Face à cela, le règne de la nature, majestueux et inépuisable, principe illimité de vie, source fécondante de tout lot de vie, ne peut être intrinsèquement vulnérable ni même fragile. Qu'on songe aux premiers mots du De rerum natura. Le livre commence par un hymne à Venus qui régente l'entière nature en plantant « le tendre amour au cœur de tous les êtres ». Cette vaste puissance amène irrésistiblement toute chose à la lumière, et de même la connaissance se doit de briser les "verrous de la nature" pour conduire l'éthique ${ }^{13}$. Deux mille ans plus tard, notre connaissance de la nature nous a ouvert ces verrous et le pouvoir technique humain s'étend jusqu'à la biosphère. Cette extension de l'action humaine à la biosphère, est depuis Günther Anders et Hans Jonas, le symbole d'une action qui n'a plus de limites, qui peut embrasser l'univers vivant ${ }^{14}$. La connaissance de la nature dont Lucrèce promettait qu'elle serait le fondement de l'éthique pose alors un nouveau problème. En permettant que l'action humaine bascule du local au global, la connaissance de la nature porte à celle-ci le plus violent des coups, et la propulse dans un autre cycle, celui de la vulnérabilité : la nature aussi peut être blessée.

Que la puissance inépuisable qui définissait autrefois la nature soit susceptible de vaciller, que la nature elle-même puisse être blessée, était quelque chose d'inconcevable pour l'Antiquité. Mais l'action de l'homme sur la biosphère brise l'impensable de toutes les époques précédentes et nous fait percevoir la nature comme étant semblable à un animal qui peut être blessé. Comment comprendre ce statut nouveau de la nature, ce pouvoir d'être blessé ? N'est-ce pas lui qui réclame notre sollicitude? Il ne faudra qu'un pas supplémentaire pour faire de la nature un vivant parmi les vivants, un supervivant, ou pour faire de la Terre un organisme (Gaïa) ${ }^{15}$. Parce que la vulnérabilité place derechef la nature au niveau de tous les autres vivants susceptibles d'être atteints dans leur chair.

Pour parer la menace d'une blessure générale de la nature, d'une Apocalypse comme ultime pas de l'agir humain, on sait que Hans Jonas a proposé une éthique de "préservation et de conservation ». Préserver et conserver, cela veut dire d'abord ne pas dépasser la limite irréversible, de ne pas outrepasser ce que la nature peut encore tolérer de notre action, ne jamais gagner la zone de l'intolérable. Autant dire qu'un trait noir borne maintenant l'horizon de notre avenir, il trace une sombre limite sur le curseur du temps, il indique la fin des temps comme désastre irréversible de la nature. Alors que la sagesse antique se donnait pour but le bien suprême, notre temps aurait plutôt pour horizon ce mal suprême, cette borne qui marque la fin des temps, l'intolérable pour la nature, ce qu'elle ne pourra souffrir davantage, la blessure mortelle. Au fond, l'heuristique de la peur proposée par Hans Jonas manifeste cette prise de conscience nouvelle au sujet de la nature : la nature elle aussi peut-être confrontée à l'intolérable, à ce qui est de l'ordre d'une blessure irréversible. On a beaucoup parlé de cette heuristique de la peur, comme moyen de préserver et de 
conserver, mais elle n'est pas l'essentiel du livre de Jonas. Le plus important est ce sentiment nouveau, ce moment fondateur d'une éthique extra-humaine qui enveloppe la vulnérabilité de la nature et de toutes des choses. Le plus important est qu'ontologie, éthique et vulnérabilité forment un nouveau thème. Faire jouer l'imagination des conséquences de nos actes, comme aiguillon d'une peur qui doit enclencher la réflexion, est une manière de donner une dimension pathétique à ce désastre possible : une nature qui ne peut plus rien tolérer. Alors que les utopies nous parlent d'un monde inachevé et postule l'espérance d'un monde achevé, mettre l'accent sur une nature qui a déjà ici et maintenant la possibilité d'être détruite en elle-même du fait de sa fragilité intrinsèque, implique tout autre chose.

17 C'est bien pourquoi il importe de prendre en considération ce qui est contenu dans le sentiment tout à fait nouveau de vulnérabilité de la nature. Quelle est son action sur nous ? À quoi nous oblige-t-il ? C'est qu'un tel sentiment n'est pas neutre. Il engendre de fait une obligation nouvelle... envers la nature elle-même. Faisons un retour en arrière pour le comprendre. Souvenons-nous que l'éthique antique ne porte jamais sur la préservation de la nature, mais sur la préservation de soi en un sens large. En effet, l'éthique grecque et latine ne concerne pas le souci de soi au sens étroit. Le souci de soi n'existe pas dans l'antiquité, malgré ce que dit Foucault dans son Histoire de la sexualité parce qu'il n'y a pas de soi qui ne soit un soi social, il n'y a qu'un soi étendu, soit dans la cité, soit dans le cosmos, à titre de citoyen de l'univers. Trouver une forme de permanence dans le changement, savoir adopter une attitude constante face aux vicissitudes de la vie, avoir une posture sociale digne est un problème pratique, le problème éthique grec et romain. Cela n'a rien à voir avec un exercice spirituel. Après avoir pensé les ruptures épistémiques de l'histoire, Foucault revient dans son Histoire de la sexualité à une continuité qui les gomme. Il trace un tableau uniforme de l'histoire comme histoire de la subjectivation qui annule l'opposition forte et bien connue entre intériorité chrétienne et extériorité antique ${ }^{16}$.

18 Nous suivons plutôt Pierre Vesperini qui réintroduit de larges coupures et restitue l'éthique romaine dans son rapport au monde. Un romain ne pense pas comme un chrétien. Il ne fait pas d'exercice spirituel. Prendre soin de soi relève pour lui d'une orthopraxie, d'un tenir droit face aux événements, et non pas d'une méditation sur soi. L'orthopraxie traite de la manière d'offrir le moins de prise à la vulnérabilité : ne pas prêter le flan aux circonstances, savoir se comporter en toutes circonstances tout particulièrement sociales, avoir une âme qui ne se laisse pas imprimer son état par le dehors. Demeurer d'aplomb, ne pas tomber, ne pas choir, toute la sémantique de la faute et de la caducité dérive de cette exigence première. Lorsque surviennent les événements plus difficiles pour soi, les plus douloureux, voire les plus terribles, comme des catastrophes, il convient de savoir rester droit. Les enseignements philosophiques ont le plus souvent eu cette fonction à Rome, enseigner à rester droit. Parce que « la vie est une guerre» (Marc-Aurèle), la philosophie doit être un guide pratique pour la traverser, une boussole pour s'orienter dans ce périple périlleux, et acquérir assez de rectitude. L'équanimité et l'égalité d'âme définissent la vertu antique romaine. L'homme vulnérable doit savoir sculpter en lui une posture impassible qui en est le corrélat, il doit répondre aux vicissitudes de la vie par l'empire sur soi, gravitas et suavitas, gravité et douceur amène ${ }^{17}$. Antonin, modèle de Marc-Aurèle aura eu ce maintien égal, ce visage serein en toutes circonstances. 
19 Par ce détour, il paraît plus évident encore que la vulnérabilité associée à la nature marque l'apparition d'une préoccupation d'un autre type qui ne concerne plus le soi dans la société et le monde. Nous sentons que l'insuffisance est portée au cœur même des choses, au sein du processus entier de la nature. La nature n'étant plus cette force de renouvellement inentamée, la voilà aussi soumise à la vicissitude des temps, à l'imprévisibilité. La vulnérabilité qui concernait les choses vivantes soumises à la désagrégation, la mort, la corruption, l'action des forces cosmiques, gagne la nature, et même la nature en entier. On prend conscience que la nature n'est pas immortelle, qu'elle aussi peut mourir et perdre sa vitalité.

C'est dans l'éclosion du sentiment de vulnérabilité de la nature que se situe la transformation de la perception de ce qui doit être protégé. Et que s'élargissent l'horizon et le site de la responsabilité humaine. Lorsque la nature entière est susceptible d'être affectée dans sa permanence même au point d'être menacée dans son existence, s'installe la conviction que la précarité n'est pas extrinsèque, événementielle, destinale ou divine, mais intrinsèque aux choses. La nature ne laisse pas s'instiller en nous seulement le sentiment de sa vulnérabilité, elle fait germer un nouvel éthos, plus complexe, celui de sa structure intrinsèquement précaire. Par un renversement complet, le règne de ce qui ne pouvait être entamé par rien, en vient à abriter en lui-même la possibilité même de sa disparition. Il n'est pas indifférent de noter que la question de la vulnérabilité de la nature est de fait devenue importante au moment précis où l'action globale de l'homme a commencé à concerner la biosphère. C'est bien parce qu'aucune époque, avant la nôtre, n'avait assisté au déploiement d'une action humaine capable de retentir sur la biosphère, la nature globale, la Terre en entier, que la question ne se posait pas auparavant. Il aura fallu que l'idéal baconien de maitrise de la nature, victime de son succès, laisse entrevoir cette perspective inouïe, non plus une nature disponible, mais une nature indisponible, qui ne laisse plus aucune disposition à l'agir, qui soustrait tout fondement à l'humain, et qui réclame à présent seulement notre sollicitude.

21 Mais revenons à notre question. Quel type d'obligation ce changement engendre-t-il ? A quoi cette situation nous oblige-telle? Envers qui ou quoi doit se manifester maintenant une forme de responsabilité ? Sans aucun doute envers la nature elle-même et pas seulement l'homme. Car, prendre en compte ce qui doit être conservé pour que l'homme perdure, c'est immédiatement dépasser la limitation anthropocentrique. Une telle obligation envers l'homme englobe de fait la nature, elle revêt un caractère totalisant. Elle porte nécessairement sur les choses « extra-humaines ». Lorsque l'agir humain embrasse la nature entière, la distinction entre nature et culture tombe aussitôt. Même en admettant que seul l'avenir de l'humanité soit en jeu, celle-ci dépend de la préservation de la nature, prémisse de l'homme dans le monde, condition d'une existence humaine, de sorte que l'obligation d'agir pour préserver l'humanité enveloppe de fait la nature. L'obligation d'une future présence de l'homme dans le monde sécrète naturellement, pourrait-on dire, cette obligation nouvelle et d'un nouveau type, qui est de prendre en charge le monde coextensif à l'homme.

L'éthique qui portait autrefois sur l'individu, le présent, l'inter-humain, s'étend maintenant à l'humanité en général, à l'avenir, à l'extra-humain en tant que nature et monde. Même si à première vue la réflexion de Hans Jonas pourrait encore paraître humaniste en son fond -en tant que la responsabilité semble porter exclusivement sur une future humanité, ou encore sur la préservation de l'existence humaine dans le 
temps, la conservation de son existence et la certitude de son avenir-, on n'a déjà plus affaire à un "exclusivisme anthropocentrique ", et donc au dogme grec-juif-chrétien, comme Hans Jonas le souligne lui-même. ${ }^{18}$ Se dirige-t-on pour autant vers une justification de la nature pour elle-même? Car après tout l'homme pourrait ne pas exister mais seulement la nature ? À première vue, ce qui préoccupe Jonas, ce n'est pas la nature en elle-même, c'est l'impossibilité de concevoir un monde sans humanité, un monde inapproprié à l'homme. Un monde qui contient l'humanité doit exister à jamais : «Qu'un tel monde doive exister à jamais dans l'avenir, un monde approprié à l'habitation humaine et que toujours à l'avenir il doive être habité par une humanité digne de ce nom, on le concédera volontiers comme un axiome universel et un but souhaitable très plausible de l'imagination spéculative $»^{19}$. Avec cette condition, « un monde approprié à l'humain", l'obligation s'élargit cependant infiniment, comme " obligation de garantir la prémisse de l'obligation ", à savoir de garantir une nature vivante, ultime condition d'une humanité vivante. Garantir ce qui rend possible la permanence d'une vie humaine sur Terre revient à poser la Terre comme objet de l'éthique.

L'obligation a beau d'abord être saisie à travers un droit qui concerne l'existence humaine, à savoir l'absolue obligation d'une existence humaine dans le présent et dans l'avenir, ce droit implique donc de préserver aussi la nature. Dans la mesure où l'humanité n'est que la pointe supérieure de la nature, c'est bien la nature entière qui est prise dans cette obligation. C'est ici que Hans Jonas nous semble le plus intéressant, dans cette connivence profonde de l'éthique et de la nature. L'humanité n'est pas à distance de la nature, elle est toujours prise dans une continuité de la nature. La communauté de destin entre l'homme et la nature se manifeste lors du plus grand danger, alors non seulement nous découvrons que nous sommes vulnérables, mais nous découvrons horrifiés que la nature est vulnérable, parce que nous comprenons aussi que la nature ne nous est pas étrangère, qu'elle nous est apparentée, nous découvrons "la dignité autonome de la nature ». Naît en nous le sentiment puissant qu'il faut la « respecter » et préserver son intégrité, que, elle aussi, est digne d'attention. La nature comme condition de l'humanité récupère une parcelle de dignité autrefois réservée à la seule humanité. La nature ne pourra plus être indifférence et absence de dignité des fins.

Chez Jonas, la continuité de la nature et la solidarité de destin de tous les êtres, humains et non humains, repose sur ce revirement complet de la pensée de la nature. Sa grande thèse est en effet de dire : toute fin est immanente à l'être, l'on ne peut séparer être et fin, dans toute la nature. Nous oblige ce qui est, nous oblige l'être qui contient ce qui doit être, cela vaut pour l'humanité, mais cela vaut pour toutes les formes de vie. C'est pourquoi le principe d'une éthique future est métaphysique et non pas éthique, il s'appuie sur l'être, sur la contrainte qu'exerce l'être : d'abord l'existence de l'humanité, mais l'existence aussi de tous les êtres de la nature en général. Jamais une éthique ancienne n'a fondé le devoir sur l'être, ici au contraire, une éthique du futur n'est possible que si elle fonde le devoir sur l'être. Jonas montre que l'être possède sa propre fin, et cela n'est possible que si l'on pense l'être à partir de la biologie et de la psychologie, contrairement à la tendance réductionniste des sciences de la nature, que si l'on pense l'être à partir du témoignage de nous-mêmes et donc de la vie. Seule la vie se pose comme souci de soi, préservation de soi, perpétuation de sa forme, ipséité contre le danger de la mort, du retour à la matière. 
Le même thème revient souvent chez Jonas, le non abandon d'une forme d'anthropomorphisme, comme signe d'inclusion à la nature ${ }^{20}$. En partant de ce qui montre dans la subjectivité personnelle mais aussi dans l'infra-subjectivité appétitive et sensitive, biologique, Hans Jonas restitue à la nature toute sa teneur ontologique. La nature récupère la capacité de poser des fins dans son être même, dans son échelle même. L'anthropomorphisme bien conduit est donc un cosmomorphisme, il dit notre appartenance à un même ordre des fins, c'est toute la nature qui s'unifie avec notre témoignage :

L'être ou la nature est un et il rend témoignage de lui-même dans ce qu'il laisse procéder de lui. Ce que l'être est, cela doit être déduit de son témoignage et naturellement de celui qui en dit le plus, du plus manifeste, et non du plus caché, du plus développé, et non du moins développé, du plus plein au plus pauvre, -donc du plus élevé qui nous soit accessible ${ }^{21}$.

Déjà Le phénomène de la vie restituait une continuité à la nature, à l'échelle des êtres, à la scala naturae. De notre conscience à la plus infime cellule, être, c'était se poser comme fin contre le non-être et la mort, contre la destruction de l'ipséité et de soi. Cette manière de se poser comme fin contre le non-être traverse toute l'échelle de la nature vivante et même, d'une certaine manière, hyperbolique, la matière. Notre subjectivité n'est pas isolée, affirme aussi le Principe responsabilité, elle est " un iceberg », elle parle au nom d'un "intérieur muet ", elle laisse deviner que l'immensité des vivants est déjà position de fins, que la vie possède des formes "pré-spirituelles", et que même la matière est déjà position de fins analogues. ${ }^{22}$ La nature est donc ce qui possède ses propre fins, pas nécessairement sous une forme subjective, mais en tout cas sous une forme déjà pré-spirituelle en toute vie, voire même dans la matière, serait-ce de manière inconsciente, souterraine. Il n'y a pas d'autre être que celui qui se pose comme fin contre le non-être. Parce que l'être, pour être, se prend nécessairement pour fin, le seul moyen pour lui de résister au non-être est de poser son être comme devant être. Autrement, cela voudrait dire qu'être est indifférent à son être, que non-être et être sont indifférents. Mais, qui peut le penser, si ce n'est un mathématicien ou un physicien qui, faisant abstraction du témoignage de sa vie, regarderait le monde comme s'il n'y avait pas des êtres devant être mais seulement des équations ou des processus matériels. Dès qu'on est, il faut abandonner toute neutralité ontologique. À moins de vouloir se repaître d'abstractions, être n'est pas une formule mathématique. Car, aucune formule ne pose une existence tandis que chaque être tend vers la préservation de son être. En prenant en charge la contrainte, pour l'être, d'être, la responsabilité prend aussitôt un caractère concret. Voilà que s'insinue dans l'être, le fait de pouvoir ne pas être, la contingence, la facticité. La précarité ne désignera plus la faiblesse humaine, ni même la vulnérabilité, elle tombera au cœur de l'être lui-même, non pas comme pouvoir ne pas être mais insuffisance radicale d'être.

Lorsque la sollicitude s'élargit à tout l'être, et non seulement à l'action épuisable, elle nous met sur la voie d'un nouveau sentiment. Hans Jonas donne pour modèle, germe, ou archétype de la responsabilité, le lien du parent à l'enfant qui vient de naître, du fait "de l'insuffisance radicale du nouveau-né", de son "extrême fragilité ». La quintessence de l'obligation réside dans le fait d'être obligé par la vulnérabilité ellemême, en l'occurrence par le nouveau-né qui l'incarne. L'humanité vulnérable à venir est dans la situation de l'enfant qui vient de naître ou qui va naître. Nous devons la prendre en charge comme nous prenons en charge le nouveau-né, du fait de sa faiblesse. L'analogie entre la faiblesse de l'humanité et la faiblesse du nouveau-né est 
d'ailleurs une thématique constante de la littérature humaniste antique et moderne. L'humanité est jetée nue dans l'existence. De Pline l'Ancien à Kierkegaard, on ne cesse de souligner la fragilité de l'existence qui commence par l'absolu dénuement et dénudement, l'exposition aux dangers de toutes sortes, sans protection, sans carapace, commencement misérable ${ }^{23}$. Mais, contrairement à la pensée humaniste, avec la natura vulnerabilis, c'est l'être lui-même qui ressemble à un nouveau-né et nous oblige par sa fragilité et sa vulnérabilité. Dès lors, l'éthique de la sollicitude concerne non seulement les humains vulnérables, l'humanité vulnérable, mais elle englobe l'être vulnérable. La nature entière, semblable au nouveau-né, est dénuée, dénudée, dépouillée, et pour cette raison aussi appelle notre sollicitude. Nous parlions de changement de perception : il réside dans ce déplacement massif de la sollicitude de l'humain vers la précarité de l'ensemble des êtres de la nature. Au-delà d'une nouvelle obligation de protection, germe alors l'idée que les êtres sont fondamentalement insuffisants en eux-mêmes du fait même de leur nature relationnelle, en un mot que les êtres sont précaires.

Ce qui m'apparaît ici le plus décisif pour cerner une ontologie de la précarité, c'est bien que la précarité ne soit plus pensée comme un état lié à la faiblesse humaine, à la fragilité humaine, tel que ce fut le cas dans la longue histoire qui va de l'Antiquité au christianisme. Elle est en son fond même, ce qui se loge dans tous les êtres. La pensée antique plaçait la faiblesse dans l'impéritie de la constitution humaine et dans l'imprévisibilité du destin. Le christianisme étendait la fragilité et la faiblesse aux choses créées, à la misérable humanité, mais aussi à l'impotence de toutes choses... La fragilité traduisait l'état originel de la créature, sa corruption physique et morale, elle était la marque de son possible non-être, de sa dépendance envers le fiat divin. Toute la création accueillait le fragile. La fragilité théologique pénétrait l'ensemble de la nature créée qui perdait sa suffisance. Ni la nature ni les choses n'étaient plus capables de se soutenir d'elles-mêmes. La fragilité humaine étant par nature théologique, elle n'était pas autre chose que l'affirmation d'une non suffisance des choses créées, que le pendant de la création, comme néant du créé. Le néo-platonisme aura su porter au plus loin les conséquences philosophiques de cette vision. Dès que l'Un ou Dieu sont placés en position éminente relativement au monde (Plotin par exemple), le monde se néantise.

Loin de ce nihilisme du monde, nous voulons au contraire pointer comment la précarité se définit par un nouveau sens ontologique. L'être est précaire, il n'est pas faible ou fragile en tant qu'il serait soumis à l'imprévisibilité du fatum ou néantisé par le divin. Précaire veut dire que tout être tient son être des relations enchevêtrées qui le constituent, qui lui sont en même temps intérieures et extérieures, relations sans lesquelles il pourrait ne pas être, et ne pourrait pas même se poser comme fin. Cette intériorité-extériorité et cet enchevêtrement, ce sont les conditions qui accompagnent tout être dans le système global de contraintes qui forme la Terre. La modernité découvre la métaphysique de la précarité qui est tout autre chose que l'histoire religieuse de la fragilité.

\section{La ruine de la nature}

$\mathrm{Au} \mathrm{XIX}^{\mathrm{e}}$ siècle, la littérature catastrophiste pensait que la ruine du monde était l'effet de la violence de la nature. Aujourd'hui, nous pensons plutôt que la ruine du monde sera l'effet de la vulnérabilité de la nature, qu'elle viendra d'une ruine de la nature elle- 
même. Anna Tsing, dans Le champignon de la fin du monde, introduit cette idée neuve, la « ruine de la nature $»^{24}$.

Que signifie "ruine de la nature »? On perçoit dans cette formule quelque chose d'inconcevable auparavant où l'on pensait plutôt aux "ruines du monde", car la nature en ruine est précisément la nature privée de sa possibilité naturante, ou qui n'a plus les ressources nécessaires pour naturer. C'est une nature sans nature, dévastée, comme un monument ancien où il ne reste qu'une esquisse de pierres. Toute la Renaissance a été imprégnée de l'imaginaire des ruines, de ce " goût " des ruines où se vivifie le sentiment d'un cycle biologique des choses $^{25}$. Dans la nature humanisée et architecturée et de la Renaissance, la ruine dit le cycle du temps, le souvenir de notre provenance, la disparition de ce qui fut édifié avec peine, longueur, fût-ce des civilisations. Esthétiquement, la poétique des ruines raccorde notre mémoire à ces temps ancestraux qui se sont taris, elle nous parle du mystère des origines, de la terrible épreuve du temps sur toute chose construite, elle nous dit à chaque fois la fin possible des mondes. C'est bien pourquoi le mot ruine ne s'applique pas à la nature: "Nous ne parlons pas de ruine à propos de fleur flétrie, de blés couchés par l'orage, où du tronc d'un arbre mort ${ }^{26}$. Que le cycle du temps affecte aussi la nature, cela, nous le savons très bien, sans nous en soucier, sans jamais dire que les saisons qui viennent ruinent les saisons qui s'effacent. Le cycle du temps peut affecter les édifices humains, il ne saurait transformer la nature en ruine d'elle-même.

Ruines humaines et ruines de la nature ne racontent donc pas la même chose, derrière ces mots se cachent des récits antithétiques : d'un côté, c'est la force destructrice du temps ou de l'homme qui rend manifeste la fragilité de toute construction humaine, d'un autre côté, c'est la force destructrice de l'art humain qui menace maintenant la nature en entier, la nature n'étant plus à l'abri de la technique, de la science, des forces transformatrices du capitalisme.

Mais, ce qu'il y a d'intéressant chez Anna Tsing, c'est de penser la nature déjà transformée en ruine d'elle-même, ce qui veut dire épuisée. Ce n'est pas une utopie dont on parle, mais le monde d'aujourd'hui, ou plutôt la fin de ce monde qui a gagné toute la surface de la Terre. Aucune catastrophe n'a été nécessaire, ni même aucun bouleversement mondial, seulement l'emboîtement infini de micro-fractures responsables de cet épuisement final.

La réflexion sur la ruine de la nature prend clairement place dans un problème plus large pour Anna Tsing, celui de la précarité. Il est intéressant de voir celle-ci étendre la précarité à l'ensemble des êtres, à la totalité du monde. Comme la vulnérabilité chez Jonas, la précarité n'est plus un état localisé, mais une situation généralisée : «La précarité est la condition de notre temps » avance Anna Tsing ${ }^{27}$, «Je considère que seule la reconnaissance de la précarité comme une condition répandue sur la surface de la Terre peut nous permettre de caractériser ce qu'est la situation du monde $»^{28}$.

Quelle est donc cette situation du monde ? Justement que la précarité ne soit plus une possibilité mais une réalité, une condition universelle. Elle ne se cantonne pas à l'homme, elle s'étend sur toute la surface de la Terre. La vulnérabilité, on l'a vu, désigne la susceptibilité à la blessure, elle renvoie les êtres à leur fragilité physique et vitale. Mais nous voulons dire plus précisément en quoi la précarité touche ici une dimension plus intime des êtres, leur épuisement. Le précaire est l'épuisé. Est épuisé, celui qui perd toutes ses relations, naturelles, sociales, économiques. Lorsque dans une situation donnée, l'appauvrissement des relations est tel que l'existence des êtres est menacée, 
alors il apparaît clairement que ces relations font partie de son être. La natura vulnerabilis était une potentialité qui ne disait pas comment elle pouvait se réaliser. La nature inépuisable de l'Antiquité, la nature vulnérable de la modernité, la nature épuisée des postmodernes, sont les jalons d'un mouvement long qui finit par priver la nature de son ressort. Le livre de Anna Tsing est foncièrement un livre sur ce dernier moment, la précarité comme épuisement vital, biologique, social, anthropologique. Inutile de recourir à l'imagination d'une Apocalypse, l'Apocalypse a lieu, elle a lieu sans cesse, continûment, c'est une Apocalypse continue (alors que l'espérance promettait une révolution continue). Par la dévastation des milieux, par l'exploitation capitaliste, se produisent inlassablement des espaces de précarité, d'instabilité, d'invivabilité, de vulnérabilité.

C'est la même chose de parler de "ruine de la nature » et d'établir que les êtres sont précaires selon des modes toujours singuliers. C'est pourquoi l'apparition de la nature ruinée est rattachée à la globalisation mais pas seulement ${ }^{29}$. Certes, il y a une condition globale, à savoir l'expansion du capitalisme qui aliène les choses et les êtres humains et non humains, en en faisant des ressources mobiles arrachées à leur tissu de relation. L'échange transnational (prix du bois, des matières premières...) consiste à changer de milieux après exploitation, dans un déplacement géographique incessant. Mais, la ruine a aussi un caractère local parce qu'elle emprunte toujours une voie d'une histoire particulière, les forêts dévastées ne sont pas les mêmes ici là, elles ont leurs histoires, racontées ici, forêts de l'Oregon, forêts du centre du Japon, forêts du Yunnan, forêts de Finlande. Chaque forêt a son histoire, relève d'un récit, d'une description.

À l'intérieur du jeu des forces globales et locales, Anna Tsing met bien en évidence que la ruine de la nature est toujours engendrée par un mécanisme d'arrachement aux relations. L'aliénation, dont elle parle, n'a rien de subjectif, de psychologique. L'aliénation désigne ce processus de destruction des relations inter-vitales au profit de l'exploitation d'une ressource mobile, processus qui rend les êtres étrangers aux uns et aux autres. La ruine de la nature est concomitante de cette destruction des relations multispécifiques, de la stérilisation des relations entre espèces. Ainsi, l'espace de vie affecté à une mono-production modifie le paysage en supprimant toute les relations vitales qui se sont nouées. Lorsqu'un tel espace de vie est ensuite abandonné parce que la ressource n'a plus de valeur, il se transforme en ruine ou milieu épuisé. Nous donnons ici ce sens à l'aliénation dont parle Anna Tsing : épuisement des possibles.

L'épuisement des possibles dévoile dès lors cet autre aspect de la précarité : que tout être est une forme de consistance, que son être est une modulation de consistance. La ruine de la nature est bien cet épuisement de possibles mais ce qui rend possible cet épuisement, c'est d'abord l'insuffisance radicale des êtres, sur laquelle il nous faut maintenant tout particulièrement insister. La prise de conscience du caractère insuffisant des êtres semble concerner aujourd'hui tous les régimes de savoir. Dire que l'être est in-suffisant, c'est dire qu'il consiste en ses relations et ne consiste qu'en ces relations. La métaphysique s'est appuyée sur la substance comme auto-suffisance, substrat invariable, mais il était déjà manifeste, qu'à part Dieu, aucune substance n'avait assez de suffisance pour se donner l'être. Que ce soit sous la forme d'une autopoïèse (biologie), d'une autoproduction (économie), d'une autonomie (éthique), d'une autarcie..., substance et forme, ces vieilles notions saisissant un être fermé en luimême, ont résisté, et résisté jusqu'à l'intérieur des sciences. Bergson notait cet attachement aux formes stables et closes jusqu'au cœur des sciences modernes. Mais 
bien des sciences nous apprennent en revanche que si les êtres manquent de suffisance, de substance, de consistance ; c'est parce qu'ils sont pris dans des réseaux de conditions multiples, larges, biologiques et évolutifs, et même liés à la géophysique ou à la biosphère. Restituer ces réseaux, c'est raconter des histoires singulières. Jusqu'ici nous avons décrit la précarité comme l'épuisement des relations. Mais encore faut-il que les êtres soient déjà compris dans leurs relations de co-dépendance, de composition, de conjugaison, de symbiose. Nous disons maintenant que les êtres sont précaires en leur fond, parce qu'ils sont impermanents, insubsistants, composés, conjugués. Être précaire, c'est être composé, et être composé, c'est consister dans un tissu de relations ou plutôt c'est toujours avoir à faire consister son tissu de relations. Les relations ne peuvent s'appauvrir que parce qu'elles constituent de fond en comble ces êtres. Possible atrophie des relations et possible monotonie (épuisement des possibles et extinction du pluriel) supposent déjà une insuffisance radicale des êtres (leur inconsistance propre) qui doit être conjurée et modulée.

Parce que les êtres dépendent d'autres êtres, ils nous racontent ainsi leur histoire de composition. On ne comprend aucun être sans les histoires qui retracent ses collaborations, ses associations, ses symbioses, ses participations entre espèces. Il est manifeste que la théorie reproductible et fermée de l'ADN, ne saurait restituer cette histoire des vivants par elle seule. Comme le suggère Anna Tsing, il faudra bien un jour rendre justice à une "théorie hologénomique du vivant ", capable de décrire la genèse de nouvelles entités non identiques, les organisme-symbiotes, les holobiotes. Le partage entre reproductibilité d'une même forme et hétérogenèse traverse les sciences biologiques et ethnographiques, remarque-t-elle. C'est pourquoi les sciences oscillent entre l'explication par des invariants ou la description qui prend en compte des histoires longues et des compositions singulières ${ }^{30}$. Expliquer ou décrire, il est impossible de choisir, les deux sont nécessaires, il faut expliquer ce qui demeure et décrire ce qui change.

Ainsi, le champignon matsutake que Anna Tsing prend pour fil de son analyse est d'abord une histoire conjuguée. Ce champignon est le corps fructifère du mycète dont la vie inter-spécifique est immense. La particularité du mycète est de réussir à digérer les pierres, raison pour laquelle plus un sol est pauvre, plus la vie des mycètes s'y propage. On a comparé à l'internet le tapis de connexions établi par les mycètes dans une forêt, "woodwide web ». Le champignon matsutake ne naît pas dans les ruines du capitalisme parce qu'il serait autosuffisant. Au contraire, il occupe cet espace en le recomposant, en recomposant la forêt. Comment comprendre la forêt en effet, sans l'activité symbiotique des vivants? Les forêts, ce sont précisément des histoires très compliquées qui manifestent des agencements momentanés, particuliers, entre agents différents, si bien que « l'espèce ne constitue pas toujours la bonne unité de base pour raconter la vie dont regorge la forêt ». Il vaudrait mieux parler de vie multi-spécifique, de polymorphie active, de symbiose d'agents. Le "sotoyama", ce paysage rural japonais, que beaucoup cherchent aujourd'hui à restaurer au Japon, repose sur une association chêne/pin/humain. Face aux effets ravageurs de la déforestation, cette association rééquilibre flores et faunes... Autant dire que «le caractère durable de la nature ne vient jamais de lui-même ", il est construit, il est aménagé, il est obtenu par des relations de consistance, par une maximisation des relations entre acteurs qui vivent d'un même écosystème ${ }^{31}$. 
41 La précarité ontologique prend donc cet ultime sens, plus précis, elle manifeste le poids de l'interdépendance, du symbiotique, de la consistance inter-êtres. En tant que tel, ce n'est pas un état passager mais le fond même des êtres. La nature en ruine et épuisée s'accompagne de cette prise de conscience : tout être est précaire parce que tout être consiste en relations qui sont autant de chaînes de conditions extérieures à lui et qui définissent son intériorité. Nous devons distinguer la précarité comme épuisement des relations et extinction de l'altérité, et la précarité comme in-consistance fondamentale des êtres. Les êtres sont toujours des processus relationnels où se créent ou non des formes de consistance dynamique, de connectivité active. C'est en ces deux sens que la modernité est hantée par le spectre de la précarité. Épuisement et modulation de l'être (in-consistance).

42 Est-ce un hasard si presque à la même époque que le livre de Hans Jonas sur Le principe responsabilité, en 1985, la question de la consistance était prise comme thème d'un séminaire de Félix Guattari, Référence et consistance ? Déjà Eugène Dupréel, dans Essais pluralistes avait montré que plus un équilibre est précaire, tout en maintenant les éléments hétérogènes et les éléments instables, plus il est consistant ${ }^{32}$. Guattari distinguera à son tour une connectivité passive des choses (référence) et une "conjonctivité active et processuelle », exprimant "la précarité, la fragilité » de l'existant. On n'a pas suffisamment insisté sur la mise en garde de Guattari : il s'agissait de remplacer les vieilles catégories substantialistes, massives, fermées, rigides, par une pensée des agencements et de la consistance de ses agencements, incluant nécessairement fragilité et précarité. On découvrait derrière les identités figées des processus de conjonction, des formes et des prises de consistance, bref on découvrait «l'être comme modulation de consistance ${ }^{33}$.

De la nature invulnérable à la natura vulnerabilis, de l'être comme substance à l'être comme modulation de consistance, de la précarité humaine à la précarité ontologique de tous les êtres, c'est bien sur ces changements notables de perception que notre pensée contemporaine s'édifie. Tous nos problèmes contemporains sont des problèmes de consistance : en quoi consistent les êtres, comment faire consister à nouveau la nature et l'homme, les vivants et la Terre sans plus les livrer à l'épuisement généralisé des relations?

Cette autre conception de l'existence doit nous retenir, elle nous impose sa logique et sa généralité : la précarité ontologique. La modernité découvre la précarité comme fond ontologique général des êtres. Pareille précarité touche tous les êtres, non en tant que créés, non en tant que soumis au destin, mais en tant que ce sont des êtres, sans aucune cause religieuse ou destinale. L'être est précaire en tant que tel, c'est-à-dire comme être, parce qu'il est être, tissé de relations qui peuvent s'épuiser et qu'il lui faut faire consister dans des processus actifs. Qu'un être, parce qu'il est être, soit en même temps précaire, est un sentiment nouveau, qui ne ressemble ni à celui de fragilité chrétienne, ni à celui de la faiblesse antique. Il ne se réduit pas non plus à notre situation sociale et économique. Il concerne l'être en lui-même. La précarité nomme autant la situation ontologique des êtres que la situation de la nature en tant que nécessaire inclusion réciproque de tous les êtres, inter-consistance. Au cœur de nombre de théories et de pratiques contemporaines, c'est cette exigence nouvelle d'avoir à décrire les phénomènes de consistance et de dé-consistance qui passe au premier plan. 


\section{NOTES}

1. J.-L. Chrétien, Fragilité, Paris, Minuit, 2017, p. 162-163. La fragilité chrétienne articule le rapport de l'humain au divin, elle n'est plus un mal seulement; elle est un remède : saint Paul, Deuxième épitre aux Corinthiens, XII, 10, « lorsque je suis faible, c'est alors que je suis fort ».

2. J. Tronto, Un monde vulnérable, Pour une politique du care, Paris, La découverte, 2009

3. M. Garrau, Politiques de la vulnérabilité, Paris, CNRS éditions, 2018.

4. P. Montebello, L'autre métaphysique, Dijon, Les presses du réel, 2015.

5. Buffon, Les époques de la nature, Supplément à l'Histoire naturelle, volume 5, Paris, Imprimerie royale, p. 225-227, 1774-1783.

6. N.-A. Boulanger, Recherches sur l'origine du despotisme oriental et des superstitions, Londres, Seyffert, 1762, p. 29.

7. Ibid., p. 26-27.

8. Ibid., p. 27.

9. H. Jonas, Le Principe responsabilité, Paris, Flammarion, 2013, p. 24.

10. Ibid., p. 26.

11. Ibid., p. 25.

12. J.-L. Chrétien, Fragilité, chapitre VII, L'élargissement chrétien de la fragilité, op. cit. p. 159.

13. Lucrèce, De rerum natura, Paris, Garnier-Flammarion, trad. par J. Kany-Turpin, 1997, p. 53 et 57.

14. H. Jonas, Le Principe responsabilité, op. cit., p. 265.

15. J. Lovelock, La terre est un être vivant, l'hypothèse Gaïa, Paris, Flammarion, 2017.

16. M. Foucault, Histoire de la sexualité, 4 volumes, Paris, Gallimard, 2014-20.

17. P. Vesperini, Droiture et mélancolie : Sur les écrits de Marc-Aurèle, Paris, Verdier, 2016.

18. H. Jonas, Le principe responsabilité, op. cit., p. 99.

19. Ibid., p. 38.

20. Ibid., p. 144-145.

21. Ibid., p. 140.

22. Ibid., p. 149.

23. J.-L. Chrétien, Fragilité, op. cit., p. 32-33.

24. A. Tsing, Le champignon de la fin du monde, Paris, La Découverte, 2017.

25. S. Forero, Le temps des ruines : le goût des ruines et les formes de la conscience historique à la Renaissance, Seyssel, Champ Vallon, 2013.

26. J.-L. Chrétien, Fragilité, op. cit., p. 80.

27. A. Tsing, Le champignon de la fin du monde, op. cit., p. 55.

28. Ibid., p. 36.

29. Ibid., p. 38 : «Les paysages de la globalisation sont aujourd'hui jonchés de ce type de ruine».

30. Ibid., p. 330.

31. Ibid., p. 27.

32. E. Dupréel, Essais pluralistes, Paris, PUF, 1949.

33. Séminaire du 05/05/87, F. Guattari https://www.revue-chimeres.fr/05-05-1987-FelixGuattari-Reference-et-consistance. 